\title{
Inégalités de santé et action territoriale: situation et perspectives offertes par les principes du développement durable
}

Jean-Marc Lorach

\section{(2) OpenEdition}

Édition électronique

URL : http://journals.openedition.org/developpementdurable/1032

DOI : 10.4000/developpementdurable.1032

ISSN : 1772-9971

Éditeur

Association DD\&T

\section{Référence électronique}

Jean-Marc Lorach, « Inégalités de santé et action territoriale: situation et perspectives offertes par les principes du développement durable », Développement durable et territoires [En ligne], Points de vue (2003-2010), mis en ligne le 05 mars 2004, consulté le 10 décembre 2020. URL : http:// journals.openedition.org/developpementdurable/1032 ; DOI : https://doi.org/10.4000/ developpementdurable.1032

Ce document a été généré automatiquement le 10 décembre 2020.

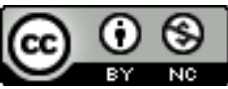

Développement Durable et Territoires est mis à disposition selon les termes de la licence Creative Commons Attribution - Pas d'Utilisation Commerciale 4.0 International. 


\title{
Inégalités de santé et action territoriale: situation et perspectives offertes par les principes du développement durable
}

\author{
Jean-Marc Lorach
}

1 A l'occasion des discussions sur le projet de loi relatif à la santé publique que le Parlement devrait adopter courant 2004, les commentateurs ont relevé que, malgré des dépenses publiques de santé parmi les plus élevées du monde, la France ne parvient toujours pas à réduire les inégalités sociales et spatiales sur son territoire.

2 Celles-ci ont même tendance à s'aggraver depuis deux décennies comme le montrait en 2002 le troisième rapport triennal du Haut Comité de la Santé Publique, où sont relevés notamment les points suivants:

3 à l'âge de 35 ans, un cadre supérieur présente une espérance de vie supérieure de 6,5 années à celle d'un ouvrier,

4 la détection du SIDA se fait après l'apparition de la maladie, soit trop tardivement pour un traitement efficace, chez $54 \%$ des patients d'origine africaine, mais seulement $21 \%$ des patients d'origine française...

5 l'espérance de vie à la naissance varie de dix ans entre les zones d'emploi du Nord et du Sud de la France,

6 le risque de surmortalité entre la trentième et la quarantième année est aggravé dans les petites villes.

7 La Déclaration Universelle des Droits de l'Homme indiquait déjà que les Etats doivent avoir pour objectif de garantir à leurs citoyens «un niveau de vie suffisant pour assurer la santé et le bien-être des individus». La Cour Européenne des Droits de l'Homme a mis en application ces principes, en légitimant en 1994 le droit à la protection de la santé des occupants d'une habitation contre les pollutions produites par une station d'épuration voisine (arrêt Lopez Ostra contre Espagne du 9 décembre 1994²). 
Ce sont à l'évidence les plus défavorisés qui cumulent les difficultés sur notre territoire, et ils sont les premiers touchés en période de restriction (hausse du forfait hospitalier et fin de l'aide médicale d'Etat). Certaines populations immigrées présentent notamment aujourd'hui d'importantes difficultés d'intégration, qui rendent souvent malaisé leur accès aux soins (Obin 1999).

9 La santé ne se réduit pas à l'absence de maladie. Elle est définie de façon élargie dans le préambule de la Constitution de l'Organisation Mondiale de la Santé (OMS), comme un état complet de bien-être physique, mental et social ${ }^{3}$. En France, le droit à un environnement sain, et donc à la santé, a été repris en avril 2003 dans la proposition de la Commission Coppens, pour lui donner valeur constitutionnelle. Mais ce droit au bien-être et à la santé, s'il est effectivement davantage reconnu, ne peut rester dans le seul champ des compétences de l'Etat. Les objectifs de développement durable affichés par la France, et par un nombre sans cesse grandissant de nos collectivités territoriales, conduisent à traiter les questions sociales à tous les niveaux de notre système organisationnel.

10 La décentralisation va dans le sens du développement durable. En effet, ce dernier concept repose notamment sur un principe tendant à privilégier l'action de proximité, confirmé lors de la Conférence fondatrice de Rio en 1992: «Think global, act local». Rappelons que le programme mondial de développement durable adopté à Rio, et baptisé Action 21 ou Agenda 21, appelait toutes les collectivités locales de la planète à se saisir du concept et à bâtir à leur propre niveau un programme Agenda 21 pour leurs territoires respectifs (Lorach et Quatrebarbes 2003).

11 Le développement durable répond en ce sens à un besoin de prise en charge au plus près des populations sur des problèmes universels. Il se situe aux antipodes d'une «économie de casino» (Ducroux, 2002). Nous examinerons, en première partie de ce point de vue, comment notre pays s'emploie déjà et à tous niveaux à lutter au plus près des populations contre les inégalités en matière de santé, en application du volet social du développement durable. Nous analyserons ensuite les limites auxquelles se heurtent cependant les politiques territoriales de lutte contre les inégalités. Cette étude se poursuivra par un point d'actualité sur les réformes législatives en cours. Notre propos se conclura enfin par une réflexion sur les pistes de mise en œuvre des principes du développement durable, susceptibles d'accroître le niveau sanitaire général du pays et de réduire durablement les inégalités en ce domaine.

12 Les Français sont très largement inégaux devant la maladie. C'est ce que démontre l'étude Eurocare $3^{4}$ qui a étudié le taux de survie de 1,8 millions d'adultes et 24000 enfants chez lesquels un cancer avait été diagnostiqué entre 1990 et 1994 (ils ont été suivis jusqu'en 1999). Selon ces données, présentées à l'occasion de la conférence européenne sur le cancer qui s'est tenue à Copenhague le 25 septembre 2003, la France s'est révélée être le pays qui présente le gradient social de mortalité par cancer le plus élevé d'Europe. En termes moins techniques, l'incidence $d u$ niveau social $d u$ malade influence spectaculairement ses chances de survie dans notre pays, alors que ce paramètre joue un rôle beaucoup moins important chez nos voisins. Il s'agit donc bien pour notre pays d'une question de développement durable, qui joue tant au niveau de la solidarité que de l'équité.

13 L'application du principe de proximité qui sous-tend le concept du développement durable nous apparaît susceptible d'apporter un début de réponse. Ce principe conduit en effet à rechercher au plus près de l'usager une optimisation globale des traitements 
apportés, plutôt que la seule réduction systématique des coûts avec ses évidents corollaires en termes d'accroissement des inégalités. Certaines politiques locales fonctionnent ainsi de façon exemplaire, au niveau des collectivités territoriales. Nous citerons en illustration les ateliers «Santé-Ville» et les Observatoires locaux de la santé.

L'organisation sanitaire française présente une multiplicité de niveaux de responsabilité, au sein desquels il n'est pas facile de se retrouver aisément (Moulinot, 2001). Le besoin d'une mise en lien des acteurs de proximité a été reconnu par nos gouvernements successifs. Le Comité interministériel des villes du 14 décembre 1999 a lancé, avec les ateliers «Santé-Ville», le volet sanitaire des contrats de ville 2000-2006. L'un des principes fondateurs du développement durable y est aujourd'hui mis en œuvre: celui de la concertation avec les parties prenantes et plus largement celui de la gouvernance (Moreau Defarges 2003). Les ateliers «Santé-Ville» permettent en effet d'associer les publics concernés à l'élaboration des stratégies opérationnelles de santé publique, en sollicitant directement l'opinion des usagers et des professionnels du secteur.

Pour ce faire, deux lignes directrices ont été tracées:

- le développement de la participation citoyenne sur l'ensemble des étapes d'élaboration des programmes territoriaux de santé publique (diagnostic, ordre des priorités, programmation, mise en œuvre, suivi),

- la plus large concertation avec les professionnels et les différentes institutions du secteur de la santé, tout comme la création et le soutien de réseaux médico-sociaux pour mobiliser et coordonner les différents acteurs en présence.

16 Dans ce cadre, sont organisées des "Conférences locales de santé», qui réunissent le public, les professionnels et le milieu associatif. Des plans d'action sont ensuite élaborés en totale concertation. Il s'agit donc bien ici de construire, à travers un processus d'association élargi, des politiques de santé qui prennent en compte les différents besoins des populations. De telles politiques ne peuvent ainsi se borner par définition à appliquer un traitement uniforme à tous, quelles que soient leurs ressources.

vement vise à un développement plus harmonieux et durable en matière sanitaire et à une lutte renforcée contre les discriminations sociales. Il s'inscrit bien dans le cadre d'une prise en charge plus directe de ces questions, au niveau territorial.

Cette initiative est renforcée par la généralisation en France des Programmes Régionaux d'Accès à la Prévention et aux Soins (P.R.A.P.S.) $)^{5}$, qui convergent avec la politique de la ville, pour réduire les inégalités encore trop souvent relevées en matière de santé publique.

19 Le suivi de ces politiques est assuré par des «Observatoires locaux de la santé» où toutes les parties prenantes sont représentées. Ceux-ci peuvent couvrir des territoires étendus, mais aussi celui d'une simple commune.

20 L'accès à la santé et le niveau des soins sont en effet loin d'être homogènes dans notre pays. La région Franche-Comté s'est ainsi employée à évaluer la distance à parcourir pour un usager qui souhaite consulter un médecin généraliste. Les chiffres sont très contrastés et montrent une grande inégalité selon le lieu d'habitation: $90 \%$ des franc-comtois trouvent ce praticien à moins de $5 \mathrm{kms}$ de leur domicile, mais $1,3 \%$ de la population doivent parcourir plus de $10 \mathrm{kms}$ pour consulter un médecin (soit 15000 personnes réparties dans 98 communes) $^{6}$.

21 Le Conseil Régional des Pays de Loire est à l'origine de la création de l'observatoire régional de la santé des pays de Loire (O.R.S.). Lancé en association avec la Direction 
régionale des affaires sanitaires et sociales (D.R.A.S.S.), c'est une association loi de 1901 dont la mission est de rassembler et d'analyser les multiples données collectées au niveau régional en matière sanitaire et sociale. L'observatoire a ouvert au public un centre de documentation, et s'emploie à informer la population et les professionnels des cinq départements de la région. Son financement est assuré par l'Etat, le conseil régional et les conseils généraux intéressés.

La ville de Martigues a créé, à son échelle et voici dix ans, l'Observatoire communal de la santé de Martigues (O.C.S), pour veiller à la bonne santé publique sur le territoire de la commune. Cet observatoire recueille ses informations auprès d'un panel de près de 2000 habitants réunis en réseau et représentant 800 postes de travail à risques. Les informations recueillies permettent de mieux connaître les différentes pathologies liées aux métiers exercés et d'aider les habitants à obtenir une meilleure indemnisation en cas de maladie professionnelle. En association avec les syndicats, le milieu médical, les associations et l'inspection du travail, l'observatoire cherche également à faciliter la prévention des risques en incitant à optimiser les postes de travail.

23 En 1662, lorsque les anglais Petty et Graunt construisirent les bases de la science démographique, ils relevaient déjà des inégalités entre citadins et ruraux en termes d'espérance de vie. Les habitants des villes souffraient en effet davantage de la famine et des épidémies que leurs compatriotes des campagnes. Ce n'est pourtant qu'au XIXème siècle que des mesures concrètes furent prises en termes d'hygiène et de santé en milieu citadin. La mise en place d'infrastructures de base, comme les réseaux d'égouts, joua un rôle efficace en termes d'amélioration de la santé publique et de réduction du taux de mortalité. Aujourd'hui, la situation s'est inversée avec une meilleure prise en charge sanitaire dans les villes que dans les campagnes. Mais les disparités subsistent. Ainsi, l'espérance de vie des cadres augmente avec la taille des agglomérations, alors que celle des ouvriers évolue encore en sens contraire. En 2001, le taux de prévalence de la tuberculose était dans notre capitale de 5 460/100 000 chez les SDF contre une moyenne parisienne de 49/100 000 et une moyenne nationale de 10/100 0007.

Les besoins se sont accrus de façon exponentielle. En 2001, les dépenses de santé ont absorbé 10,1\% du PIB français, soit 148 milliards d'euros et une moyenne de 22437 euros par habitant (dont $90 \%$ sont pris en charge par la collectivité publique) ${ }^{8}$. Notre système national d'assurance maladie aura accumulé 30 milliards d'euros de déficit entre 2001 et 20049. La question se pose donc aujourd'hui de davantage responsabiliser nos compatriotes pour tenter de freiner la croissance de ces dépenses, mais en évitant de désolvabiliser les plus pauvres. L'Etat joue ici un rôle essentiel en matière de péréquation sociale. Or notre pays est actuellement engagé dans un profond mouvement de décentralisation, qui vise à donner un pouvoir sans précédent aux collectivités territoriales. Au $1^{\mathrm{er}}$ janvier 2005, lorsque la réforme aura pris toute son amplitude, près d'un point du PIB national aura été transféré du budget de l'Etat à celui des collectivités territoriales, soit 13 milliards d'euros, tandis que 1200000 fonctionnaires de l'Etat devraient avoir rejoint le million de fonctionnaires déjà employés directement par les collectivités territoriales selon les chiffres indiqués par le Président de l'Association des régions de France et le Président de l'Association des départements de France ${ }^{10}$.

Cette réforme va donc restructurer en profondeur le paysage public français, et conduit nécessairement à un retrait progressif de l'Etat. Celui-ci entrânera rapidement une montée en force accrue des collectivités territoriales, en matière de santé publique, avec en corollaire une possibilité de différenciations locales davantage contrastées. 
L'évolution n'est pas facile, car notre système est lourd: le désengagement de l'Etat doit prendre en compte un empilement de strates structurelles qui complexifient l'action publique. Divers niveaux se superposent aujourd'hui et parfois se chevauchent dans une accumulation qui nuit à la lisibilité: syndicats à vocation unique ou multiple, communautés de communes, communautés d'agglomérations, communautés urbaines, pays, cantons, départements, régions (ces dernières figurant aujourd'hui constitutionnellement au rang des collectivités territoriales de la République )...

Il conviendra de veiller à ce que le mouvement en cours de transfert de compétences de l'Etat n'aille pas, par certains côtés, à l'encontre de l'intérêt général. Tout en débouchant sur une meilleure prise en compte des réalités locales, il doit aussi préserver la cohérence et l'efficacité, gages d'un traitement homogène du corps social dans l'accès aux soins selon un bon niveau de qualité générale.

S'ajoutent aux difficultés induites par cette stratification territoriale, divers éléments susceptibles de diminuer les solidarités existantes. Parmi ceux-ci, figurent notamment les suivants:

- Risque d'un accroissement des inégalités sociales, à travers d'éventuelles politiques locales de réduction des prises en charge publiques au bénéfice des assurances privées, dont la vocation n'est pas d'assurer un service bénévole,

- Risque d'une augmentation des disparités spatiales entre territoires riches et pauvres. Il est, par exemple, impossible d'aligner l'Ile de France et le Limousin en se basant sur leurs seules ressources financières, compte tenu de l'implantation contrastée des entreprises et des hauts revenus sur leurs territoires respectifs ${ }^{11}$.

- Risque de traitements différenciés selon les populations demanderesses, comme le souligne un rapport piloté par le Centre de recherches sur les enjeux contemporains en santé publique (CRESP) et remis aux pouvoirs publics en mars $2002^{12}$.

Par ailleurs, les territoires rencontrent aujourd'hui encore des difficultés importantes pour mettre en place des stratégies volontaristes de développement durable, par exemple dans la lutte contre l'habitat indigne. Rappelons à ce titre que la loi S.R.U. du 13 décembre 2000 a institué un critère de $20 \%$ de logements sociaux (Rollot 2002), qui sont loin d'être encore concrétisés sur le terrain. Nombre de municipalités «aisées» ont marqué leur réticence à ancrer ainsi durablement le voisinage et l'électorat correspondants. A l'opposé et dans les cités défavorisées, la lutte contre le saturnisme dans les bâtiments scolaires se confronte aujourd'hui à l'absence d'une réponse collective à des problématiques ciblées. Selon une étude, publiée en janvier 1999 par l'Institut National de la Santé et de la Recherche Médicale (INSERM), 250000 enfants âgés de moins de 6 ans étaient exposés en France à des plombémies anormales du fait de leur évolution dans des environnements à risque ${ }^{13}$. Force est de constater aujourd'hui que ce sont bien souvent les communes les moins riches qui ont les besoins les plus pressants.

L'actualité législative est aussi dense que contrastée.

Les députés ont commencé le 23 février 2004 l'examen en première lecture du projet de loi sur les «responsabilités locales», qui transfère notamment l'action sociale de l'Etat vers les départements. L'actualité législative est aujourd'hui particulièrement dense dans les domaines étudiés. Nous citerons notamment en ce sens plusieurs textes particulièrement importants:

- Le projet de loi relatif à la politique de santé publique fixera le cadre des programmes de santé pour la période 2004-2008. Il a été adopté par le Conseil des Ministres du 21 mai 
2003 et a fait lui aussi l'objet d'une large concertation avec l'ensemble des parties prenantes.

Ce projet de loi a pour objectif affiché de réduire les inégalités excessives relevées en France en ce qui concerne l'état de santé de la population dont les taux de mortalité prématurée et de mortalité évitable sont jugés trop élevés. Le texte traduit la volonté affichée par l'Etat, les collectivités territoriales et l'immense majorité des acteurs, de simplifier notre système de santé. Tous s'accordent en effet à dénoncer sa complexification à l'extrême, qui s'est faite au détriment de l'efficacité et de la cohérence de l'action publique. Le projet de loi vise en ce sens à clarifier les compétences respectives de l'Etat, des collectivités territoriales et des autres entités en charge de la santé publique. Cette politique passe par la fin de la concentration excessive des moyens sur les soins et leur régulation. Les outils préconisés correspondent à ceux du développement durable: réduction des risques en amont, information et éducation des publics concernés, développement des actions de proximité.

- En ce qui concerne la réforme de notre système de sécurité sociale, une première synthèse du Haut Conseil de l'Assurance Maladie a été remise en janvier 2004 au Ministère de la Santé. Le Haut Conseil y préconise notamment une meilleure articulation $\mathrm{du}$ travail des différents acteurs au service du patient et sa meilleure répartition sur les territoires, à travers notamment une réorganisation du système sanitaire (généralistes, spécialistes, hôpitaux, cliniques...). En février 2004, une phase de dialogue, de concertation et de négociation a été engagée par les pouvoirs publics. Le processus adopté s'intègre à ce titre aux principes du développement durable. Les différentes parties prenantes ont déjà souligné leur attachement au maintien d'un système solidaire, obligatoire et universel où chacun contribuera en fonction de ses moyens et recevra selon ses besoins ${ }^{14}$. Un document d'orientation sur la réforme devrait en être issu au mois d'avril 2004.

- Deux projets de décrets relatifs à la réforme de l'aide médicale d'Etat (AME) viennent par contre de se heurter à un avis défavorable de la Caisse Nationale d'Assurance Maladie. Le nouveau dispositif de l'AME concerne les modalités de la prise en charge des soins des étrangers en situation irrégulière dont les revenus mensuels ne dépassent pas 566 euros. Ces projets de décrets sont apparus relativement complexes et trop lourds dans l'énumération des pièces justificatives réclamées aux étrangers pour accéder aux soins (preuves de l'identité, de la domiciliation, du montant des revenus...). Le risque relevé par les administrateurs de la CNAM est d'aboutir à rendre illusoire l'accès aux soins pour la majorité de la population concernée et de les maintenir ainsi dans une situation de précarité et de fragilité sanitaire. Le monde politique n'est pas resté indifférent à cette controverse. Claude Pigement, délégué national du PS à la santé, a demandé au gouvernement de tenir compte de cet avis même s'il est consultatif. Dans le même temps, Marie-Anne Montchamp, députée UMP du Val de Marne, a demandé la suspension de la parution de ces décrets. La réforme de l'AME a cependant déjà commencé à s'appliquer avec la mise en œuvre de plusieurs mesures qui étaient d'application immédiate, et n'avaient pas besoin de décrets pour ce faire. Ainsi depuis février 2004, des demandes d'AME ont déjà été refusées sur la base de la règle aujourd'hui applicable d'une résidence continue sur le territoire français pendant les trois derniers mois. En ce sens, Médecins du Monde relève que des demandeurs se sont vus refuser l'AME, parce qu'ils n'ont pu produire en temps utile les pièces justificatives de résidence alors qu'ils habitaient concrètement depuis plusieurs années dans notre pays ${ }^{15}$. 
- Le Parlement pourrait, par ailleurs, adopter au premier semestre 2004 les suites législatives du plan «Vieillissement et solidarités», présenté par le gouvernement de JeanPierre Raffarin en novembre 2003. Ce plan fait partie des mesures prises après la canicule de l'été précédent, et annonce diverses mesures dont notamment un programme d'ouverture de 100000 lits médicalisés pour les personnes âgées d'ici 2015, un plan de climatisation des maisons de retraite ou encore un programme spécifique en faveur des personnes handicapées avec «un droit à une compensation personnalisée des handicaps». Le projet de loi pour «l'égalité des droits des personnes handicapées» de Marie-Thérèse Boisseau, Secrétaire d'Etat chargée des handicapés, prévoit en ce sens un droit à compensation des handicaps qui serait soumis notamment à des conditions de ressources. Il marque une avancée mais est encore jugé insuffisant en l'état par les sénateurs ${ }^{16} e t$ les associations de personnes handicapées. Ces dernières souhaiteraient notamment une prise en charge de la totalité des surcoûts liés au handicap, et que cette prise en charge relève de la protection sociale et non de l'aide sociale ${ }^{17}$. Il est prévu de sécuriser et pérenniser l'Allocation Personnalisée d'Autonomie (A.P.A.), créée par le gouvernement de Lionel Jospin, qui risquait d'être victime de son succès. L'A.P.A. est en effet un bon exemple de compétence territoriale exercée utilement dans une logique de proximité à travers la mise en lien des différentes collectivités compétentes avec les acteurs directement intéressés: le dossier est constitué auprès du CCAS ${ }^{18}$ de la commune de résidence qui le transmet au Conseil général. Après évaluation par les équipes médicosociales en relation avec le médecin traitant de la personne âgée, un plan d'aide est soumis pour acceptation à la personne âgée et à son entourage. Le dossier est enfin présenté à la commission départementale compétente qui fixe le montant de l'aide en fonction du plan d'aide établi et du ticket modérateur.

Les avis sont donc contrastés sur les réformes en cours dans le domaine sanitaire. Le Conseil Economique et Social s'est auto saisi de ces questions depuis juillet 2003. Le rapporteur de sa section des affaires sociales s'est montré relativement critique au regard de la pluralité de textes en présence. Il estime en ce sens que «c'est à une refondation profonde du système qu'il aurait fallu s'attaquer», en relevant un manque de cohérence des politiques en cours qui segmentent notamment les réformes de l'allocation pour l'autonomie (APA), de la Sécurité Sociale, de la loi pour les personnes handicapées, des collectivités territoriales. Il précise: «Si on révisait l'ensemble du système, on pourrait éviter des coûts supplémentaires» ${ }^{19}$.

Le cadre législatif va cependant nécessairement évoluer dans un sens qui apparaît davantage conforme aux préceptes du développement durable et à une plus grande relation de proximité. Le rôle que jouait l'Etat en termes d'équité et de péréquation devra être, dans ce cadre, pérennisé sous une forme ou une autre. Examinons à présent quelles sont les pistes de réflexion sur lesquelles la mise en œuvre en France du développement durable irait dans le sens d'une lutte plus efficace contre les inégalités relevées en termes de santé publique.

Le juriste assiste aujourd'hui à l'émergence d'une génération de droits nouveaux, où le droit à la santé quel que soit le niveau de revenu, comme le droit à une eau ou à un air purs, viennent se placer au même rang que le droit au travail ou à la protection des libertés individuelles. La mise en œuvre des principes du développement durable devrait donc permettre d'avancer vers une couverture sanitaire plus homogène des populations vivant sur notre territoire. C'est ainsi que les différents principes, qui forment le substrat du concept, présentent un éventail d'outils tout à fait intéressant. 
40 Dans l'esprit qui a présidé à la création des ateliers Santé-Ville ${ }^{20}$, il conviendrait de généraliser sur chaque territoire la mise en œuvre d'une réelle pratique de concertation, permettant d'associer les différentes parties prenantes du système de santé: usagers et professionnels bien sûr, mais également secteur associatif, syndicats et institutions compétentes. La programmation, la mise en œuvre et le suivi des plans d'action sanitaire devraient nécessairement être issus de cette concertation afin d'optimiser la réponse aux demandes des différentes composantes du corps social. En ce sens, il apparaît utile d'élargir le mouvement de concertation «usagers-professions de santé-élus locaux» amorcé à travers les Schémas Régionaux d'Organisation Sanitaire depuis 2003, et de faire une large place à la médecine libérale dont l'action de proximité reste irremplaçable pour des services en demande croissante, comme l'hospitalisation à domicile. Cette version démocratique et participative d'une santé communautaire serait ainsi la déclinaison sectorielle d'un principe fondateur du développement durable, induisant des relations plus égalitaires entre les autorités, les professionnels et leurs patients.

41 La Stratégie nationale de développement durable, annoncée au printemps 2003 par le gouvernement de Jean-Pierre Raffarin, comprend un utile volet de sensibilisation des populations. Dans cet esprit, il semble opportun d'encourager au plan local l'information en matière d'hygiène et de santé publique. Celle-ci vise bien-sûr le milieu scolaire, mais aussi nécessairement les adultes à travers des actions ciblées. Toutes catégories de population confondues, la prévention apparaît essentielle; une telle politique ne peut en effet qu'améliorer l'état sanitaire d'un pays où la mortalité prématurée des hommes de moins de 65 ans est l'une des plus élevées d'Europe (suicides, accidents de la route, consommation excessive de tabac et d'alcool... ${ }^{21}$. Il est également patent que les populations les plus défavorisées sont celles qui sont les moins informées en matière sanitaire. En sont la preuve leur niveau élevé de tabagisme et une incompréhension souvent observée devant les objectifs poursuivis par la hausse des taxes sur les cigarettes. De même, la prévention du SIDA doit se faire de façon ciblée en direction des immigrés de première génération qui sont aujourd'hui parmi les plus touchés. C'est en effet également sur ces populations souvent délaissées que le développement durable amène à porter localement l'effort.

42 L'éthique se reformule aujourd'hui autour du principe de responsabilité (Jonas, 2001). La responsabilisation sociale ou sociétale est un thème d'actualité et les activités génératrices de pollutions doivent en assumer naturellement les conséquences. Nombre d'élus en ont déjà pris conscience (Follenfant, 2003), mais il y également nécessité d'une responsabilisation du citoyen. L'application raisonnée de ce principe conduit ainsi à demander une plus grande participation financière sur les dépenses dites «de confort». Elle ne doit pas cependant dériver vers l'institutionnalisation d'une santé à deux vitesses, qui serait par essence contraire aux principes fondateurs du développement durable.

43 D'un autre côté et dans une optique d'émulation, il serait intéressant de réfléchir à la mise en œuvre d'un nouveau système d'organisation des moyens de prévention, de diagnostic et de soins, qui permette aux collectivités territoriales et à l'Etat de relier davantage l'efficacité médicale aux financements engagés. Les centres de soins les plus performants pourraient par exemple recevoir des financements additionnels qu'il leur serait possible de réinvestir ensuite dans une logique de progrès continu, dans les limites nécessairement posées par la solidarité nationale.

44 En conclusion de ce point de vue, l'évolution des impératifs de santé sur des territoires fortement disparates conduit donc bien à réexaminer nos politiques de santé publique, en 
s'appuyant sur la nouvelle grille de lecture offerte par le développement durable. L'application des principes qui gouvernent ce concept pourrait en ce sens conduire à un «nouveau contrat social», présentant davantage de gages de solidarité, et où chacun serait nécessairement responsabilisé dans le sens d'une meilleure prise en charge sanitaire et de la réduction durable des inégalités.

\section{BIBLIOGRAPHIE}

BECK U., La société du risque, sur la voie d'une autre modernité (ISBN: 2080800582), Paris, Flammarion, 2003, 522 pages

FOLLENFANT T., Développement durable: 21 maires s'engagent (ISBN: 2749101190), Paris, Le Cherche Midi, 2003, 236 pages

DUCROUX A.-M., Les utopistes du développement durable (ISBN: 2746702304), Paris, Autrement, 2002, 342 pages

JONAS H., Le principe responsabilité (ISBN: 20808140208), Paris, Flammarion, 2001, 470 pages

LORACH J.-M., QUATREBARBES E., Le Guide du territoire durable (ISBN: 2744060364), Paris, Village Mondial, 2003, 416 pages

MOREAU DEFARGES, La Gouvernance (ISBN: 2130537456), Paris, Presses Universitaires de France, 2003, 127 pages

MOULINOT D., Organisation de la santé et de l'action sociale (ISBN: 2216090387), Paris, Foucher, 2001, 128 pages

OBIN J.-P., Immigration et intégration (ISBN: 2011706114), Paris, Hachette, 1999, 127 pages

ROLLOT J.-M., La loi SRU et le nouvel urbanisme communal (ISBN: 2905529229), Paris, Sorman, 2002, 240 pages

\section{NOTES}

1. Troisième rapport triennal du Haut Comité de la Santé Publique (HSCP), La santé en France 2002, La Documentation française 2002.

2. CEDH 9 décembre 1994, Lopez Ostra c/ Espagne, série A n³03-C, JCP 1995, G, 1, 3823, chron. F. Sudre.

3. Les Etats membres de l'OMS ont réaffirmé en mai 1998 leur attachement à ce que la santé figure au rang des droits fondamentaux.

4. Les études Eurocare ont été menées depuis plus de 10 ans à travers toute l'Europe. Elles rassemblent des données sur le cancer dans 22 pays et couvrent 42 types de cancer.

L'étude Eurocare 3 a été baptisée «Survie au cancer en Europe à la fin du XXème siècle» ; elle a été réalisée sous l'égide de l'International Agency for Research on Cancer (I.A.R.C.), agence spécialisée de l'Organisation Mondiale de la Santé (cf. International Agency for Research on Cancer, M. P. Coleman, G. Gatta, A. Verdecchia, J. Estève, M. Sant, H. Storm, C. 
Allemani, L. Ciccolallo, M. Santaquilani, F. Berrino, and the Eurocare Working Group, Eurocare-3: cancer survival in Europe at the end of the 20th century, Ann. Oncology 14: v128-v149 / dec. 2003)

5. Les P.R.A.P.S. ont été institués par l'article 71 de la loi n 98-657 du 29 juillet 1998 relative à la lutte contre les exclusions.

6. C. Bessin, D. Carel, V. Lucas-Gabrielli, F. Tonnelier, «Villes, campagnes, régions, Europe...», ADSP n²9, décembre 1999.

7. Ces données sont issues d'une opération de dépistage menée à Paris par le SAMU Social, qui a mis sur pied une mission dédiée à la lutte contre la tuberculose. Cette opération de dépistage a été réalisée courant 2000 sur un échantillon de 586 personnes sans domicile fixe (cf. interview de Xavier Emmanuelli, président du SAMU Social, dans Le Figaro du 21 mars 2001).

8. Chiffres annoncés par le Ministère de la Santé le 5 septembre 2002 au sortir de l'examen des comptes de la Nation.

9. Source: Comptes de la Sécurité Sociale 2003; chiffres repris à l'occasion de l'installation le 13 octobre 2003 par le Premier Ministre du Haut Conseil de l'Assurance Maladie, présidé par Bertrand Fragonard.

10. Gérard Longuet et Jean Puech, in Le Figaro du 9 octobre 2003.

11. En 2000, le PIB annuel par habitant était respectivement de 35946 euros en Ile-deFrance et de 18859 euros dans le Limousin. Quant au PIB annuel total de ces deux régions, il représentait en Ile-de-France $28,6 \%$ du PIB national et pour le Limousin seulement 1,0\% du même PIB national (source: INSEE - Comptes régionaux).

12. Rapport remis en mars 2002 à la Direction de la population et des migrations du Ministère de l'emploi et de la solidarité / étude CRESP, INSERM EPI 9905 et Université Paris XIII (Fassin D., Carde E., Ferré N. et al. Un traitement inégal. Les discriminations dans l'accès aux soins. Bobigny: Rapport d'étude $\mathrm{n}^{\circ} 5$, CRESP 2002).

13. Le Monde du 15 janvier 1999.

14. Le Figaro du 14 janvier 2004

15. Rejets de demande d'AME, relevés par Chantal de Casablanca, de la mission Médecins du Monde à Genevilliers ( Hauts de Seine ), in Le Monde du 26 février 2004.

16. La Commission des affaires sociales du Sénat a déposé 103 amendements.

17. Déclarations de Marie-Sophie Desaule, présidente de l'APF (Association des Paralysés de France) et de Laurence Tiennot-Herment, présidente de l'AFM (Association de Lutte contre la Myopathie), in Le Figaro du 24 février 2004.

18. Centre Communal d'Action Sociale. Ces centres ont été créés en 1986 pour gérer l'aide sociale, en succession des B.A.S. (Bureaux d'Action Sociale).

19. Rapport rendu au CES par Maurice Bonnet, rapporteur de la section des affaires sociales - Conseil Economique et Social, février 2004.

20. Le Comité Interministériel des Villes du 14 décembre 1999 a fixé les conditions de mise en œuvre du volet santé des contrats de ville 2000-2006 s'appuyant sur les ateliers «Santé-Ville».

21. Source: Troisième rapport triennal du Haut Comité de la Santé Publique ( HSCP ) cité plus haut, La santé en France 2002, La Documentation Française 2002. 


\section{RÉSUMÉS}

Malgré des dépenses de santé très élevées, la France connaît de fortes inégalités sociales et spatiales sur son territoire en matière sanitaire. La mise en œuvre des principes du développement durable présente l'opportunité de développer de nouvelles politiques de santé publique au plan local, susceptibles de réduire les inégalités relevées.

Despite significant spending on health, France is affected by important social and regional inequalities with respect to questions of public health. The application of the principles of sustainable development presents the opportunity to develop new public health policies at the local level. These policies are likely to reduce these social and regional inequalities.

\section{INDEX}

Keywords : sustainable development, health, inequality, poverty, territory

Mots-clés : territoire, inégalité, pauvreté, santé

\section{AUTEUR}

\section{JEAN-MARC LORACH}

Docteur en Droit, CPA-HEC, IEP et CAPA, Jean-Marc Lorach a été Chef de cabinet dans un service du Premier Ministre puis Directeur général d'une filiale d'EDF. Il est Vice-Président du Centre d'Etudes Secteur Public Secteur Privé, et enseigne le développement durable et le droit social à l'université Paris XII et en 3ème cycle de management. Dernières publications: Les maires face au développement durable, La Tribune, 12 septembre 2002 ; Le guide du territoire durable, éditions Village Mondial, janvier 2003 ; Le nouveau régime de l'élu local, éditions du Puits Fleuri, mars 2003 ; Recours et voies d'action du citoyen face à la collectivité locale, éditions du Puits Fleuri, novembre 2003 